
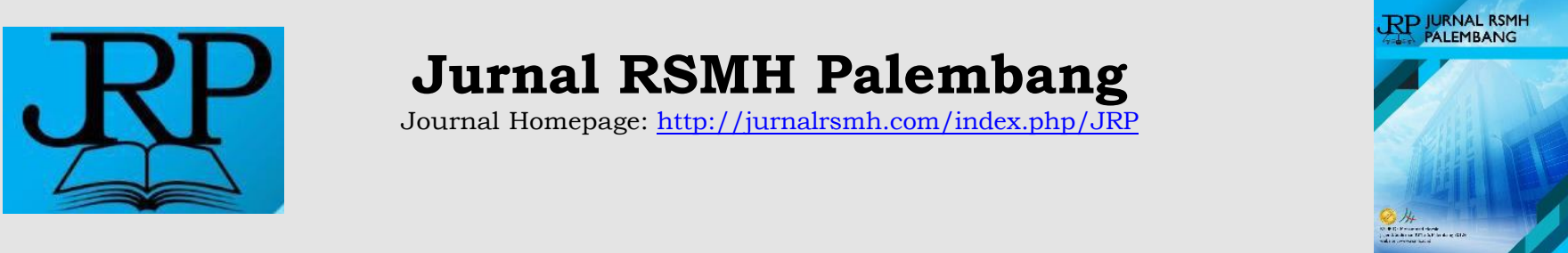

\title{
Effectiveness Of Ranitidine Providing Compared With Omeprazol In Children With Gastroesofageal Reflux Disease
}

\author{
Rizki Nandasari Sulbahri, Hasri Salwan, Raden Muhammad Indra, Sri Kesuma Astuti.
}

Medical Staff Group of Children, Faculty of Medicine, Sriwijaya University-RSMH, Palembang

\section{A R T I C L E I N F O}

\section{Keywords:}

Proton Pump Inhibitors (PPI)

Histamine H2 Receptor Antagonists (H2 RA)

Gastroesophageal Reflux Disease (GERD)

\section{Corresponding author: \\ Hasri Salwan \\ E-mail address: \\ hasrisalwan@gmail.com}

All authors have reviewed and approved the final version of the manuscript.

https://doi.org/10.37275/JRP.v1i1.1

\begin{abstract}
A B S T R A C T
Background. The drugs that are often given to children with GERD are stomach acid suppressants, namely the $\mathrm{H} 2$ receptor antagonist and proton pump inhibitor (PPI) class of drugs, but the effectiveness of the two drugs is still controversial. Objective. To evaluate the use of PPIs and H2 RA in children with GERD through evidence-based case studies. Methods. Systematic search for literature using the search instrument PUBMED, Cochrane, Google Scholar, Pediatrica Indonesiana, and Sari Pediatri. Searches included systematic review articles, randomized controlled clinical trials and cohort studies. Abstract only studies, non-clinical evaluation results, and case reports were excluded. Results. The study was obtained from three RCT studies comparing the effectiveness of omeprazole and ranitidine in the treatment of GERD, all of which have differences. Azizollahi et al demonstrated that after 2 weeks of standard doses of omeprazole or ranitidine there was a comparable significant improvement. Ummarino et al demonstrated that omeprazole was significantly better than high-dose ranitidine. Cucchiara et al (1993) showed that high doses of ranitidine were as good as omeprazole. Another study by Pfefferkorn et al showed no significant effect on the addition of omeprazole therapy combined with ranitidine in preventing the incidence of NAB. A study by Boccia et al comparing omeprazole, ranitidine, and non-therapy, found very low relapse rates. Conclusion. Evidence regarding the use of ranitidine versus omeprazole in infants and children is lacking. Based on one study specifically in the infant age group, omeprazole and ranitidine were of comparable effectiveness. A higher dose of ranitidine may have a better effect. In terms of complete symptom relief, omeprazole is likely to be superior to ranitidine.
\end{abstract}

\section{Introduction}

Gastroesophageal reflux (RGE) is a frequent phenomenon characterized by involuntary passage of gastric contents into the esophagus with or without regurgitation or vomiting. ${ }^{1}$ At $2-3$ months of age this usually occurs in the form of regurgitation of milk after drinking. Most of this reflux is confined to the distal esophagus, is brief and asymptomatic. These symptoms will disappear on their own with age. ${ }^{1,2}$

The definition of gastroesophageal reflux disease (GERD) in children is a pathological RGE phenomenon that is associated with mucosal damage and disrupts the child's quality of life and even causes growth failure. This condition is a chronic disease, its prevalence is estimated at $18.1 \%-27.8 \%$ in North America, 8.8\% -25.9\% in Europe, and 7.8\% -8.8\% in East Asia. The incidence of GERD is estimated to be around 5 per 1000 people per year. ${ }^{3}$ The pathological reflux can cause severe symptoms, interfere with quality of life and cause complications. Pathological reflux has a higher frequency, duration and severity than physiological reflux. GERD is more often manifested by regurgitation (especially postprandial), signs of esophagitis (irritability, arching position, choking, refusal to eat) ${ }^{4}$

Diagnosis is done to establish GERD are clinical symptoms, endoscopic examination, monitoring of 
esophageal $\mathrm{pH}$ and biopsy examination to see histology.

Symptoms associated with GERD in infants and children include general symptoms, gastrointestinal symptoms, and respiratory symptoms. Complications of GERD include impaired growth to failure to thrive, hematemesis, reflux esophagitis and esophageal Barrett. 4,5

Management of GERD requires a long period of time consisting of non-pharmacological and pharmacological therapy. Medical management for children includes (i) changing the viscosity of foods with alginates; (ii) changing gastric $\mathrm{pH}$ with antacids, histamine $\mathrm{H} 2$ receptor antagonists or proton pump inhibitors and (iii) altering intestinal motility with prokinetics, such as cisapride, metoclopramide and domperidone. ${ }^{5}$ Drugs that are often given to children with GERD are gastric suppressants. namely the class of $\mathrm{H} 2$ receptor antagonists and proton pump inhibitor (PPI) drugs. The indications for drug selection and the long-term safety of the two groups are controversial. Several studies have shown that both groups of drugs are equally effective. ${ }^{6}$ There is a barrier that none of the omeprazole preparations in RSMH is in the form of a suspension, making it difficult to use in infants and children. Therefore, this evidence-based case presentation aims to determine the effectiveness of administering ranitidine compared to omeprazole to treat pediatric patients with GERD.

\section{CASE ILLUSTRATION}

A boy aged 5 months with complaints of laziness to breastfeed, often fussy, and often hears grok-grok breaths, especially after giving milk. There were no complaints of vomiting, breathlessness and no blue. The patient initially presented with blisters and peeling skin complaints, the patient was treated and diagnosed with epidermolysis bullosa and poor nutrition. Currently, topical treatment and nutritional care have been obtained by giving milk via a nasogastric tube. Complete basic immunization history. General physical examination includes compost mental awareness, heart rate $110 x / m$, respiratory rate $30 x /$ minute, temperature $36.7^{\circ} \mathrm{C}, \mathrm{SpO}_{2} 98 \%$. The nutritional status of the patient was malnutrition of normal stature with a body weight of $3.5 \mathrm{~kg}$ (<-3 SD (underweight), $61 \mathrm{~cm}$ high (-2 SD to 0 SD (normoheight). Weight according to height $<-3 \mathrm{SD}$ (severely) wasted) based on the WHO curve.

Special examination showed that the conjunctival eyes were not pale, the sclera was not icteric, the face was like an old man. Chest examination showed that there were ribs, heart and lung within normal limits. Abdominal examination found flat, limp, normal bowel sounds, liver and spleen were not palpable. Warm acral limb, CRT $<3$ seconds. Dermatologic status in the colli region, posterior trunk, right thoracic, right abdomen, right femoral, right cruris, and left human $V$ digit: there were multiple irregular erosions, multiple numular hypopigmented patches, partially confluent discrete plaques.

Laboratory tests showed Hb 9.8 g/dL, MCV $68.5 \mathrm{fl}$, $\mathrm{MCH} 21 \mathrm{pg}, \mathrm{MCHC} 31 \%$, platelets $1.270 .000 / \mathrm{mm}^{3}$, LED $34 \mathrm{~mm}$ / hour, transferrin saturation 16.4\%, albumin $2.2 \mathrm{mg} / \mathrm{dl}$. The results of peripheral blood imaging showed that microcytic hypochromic anemia was accompanied by an inflammatory process. On routine urinalysis and feces the impression is within normal limits.

The results of the fiber optic laryngoscope examination performed by the ENT division showed that the epiglottis looked stiff, collapsed in the cuneiform cartilage and shortened the ariepiglottic folds, white plaques appeared on the epiglottis, arytenoid, and periform sinus. The movement of the vocal cords and ventricles has not been assessed. The impression from the examination shows laryngomalacia grade II-III dd / primary laryngeal TB. Patients also have symptoms of frequent fussiness and restlessness, accompanied by difficulty in feeding and weight gain difficulty. Based on the alarm signal from Rome's criteria, the diagnosis of gastroesophageal reflux disease can be confirmed in this patient in the form of complaints of fussiness, anxiety, frequent crying, eating problems and failure to thrive. The GERD 
questionnaire scoring is said to lead to GERD if the score is above 9 , in this patient a score of 12 . However, the patient has not performed diagnostic support tests, namely endoscopy and $\mathrm{pH}$ metri. Patients were given intravenous omeprazole therapy at first, then in the planning of oral therapy omeprazole suspension was absent while granules from omeprazole capsules were difficult in its application. Consideration of $\mathrm{H} 2$ antihistamine antagonist therapy, ranitidine suspension in patients.

\section{CLINICAL PROBLEMS}

Various studies have been conducted to determine the effectiveness of anti-secretory drugs in cases of children with GERD. The case illustration shows a patient aged 5 months with the problem of frequent fussiness, restlessness, lazy breastfeeding and often heard groggy breath after giving milk. This leads to a diagnosis of GERD. Patients are given anti-secretory drug therapy, but the availability of proton pump inhibitor class drugs, in this case omeprazole suspension, is not available, causing its administration to have obstacles. We must look for other drugs that can be given. The administration of ranitidine suspension can be considered in these patients.

Based on the introduction and the existing cases, the following questions arise: How effective is the administration of ranitidine compared to omeprazole in children with gastroesophageal reflux disease (GERD).
Q: Gastroesophageal reflux disease in children

I: Ranitidine

C: Omeprazole

O: Improvement of GERD symptoms

\section{Method Reseacrh}

The literature search procedure to answer the above problem is to search the literature online using an electronic database (PubMed, Google Scholar, Sari Pediatri, Pediatrica Indonesiana and Chocrane) with keywords: "reflux oesophagitis $O R$ esophagitis OR GERD OR gastroesophageal reflux disease OR gastroesophageal reflux OR reflux esophagitis ", " Children OR pediatric ", " Proton pump inhibitor OR omeprazole ", “Antihistamine $\mathrm{H} 2$ $O R$ ranitidine antagonists ", for searches in English and" Reflux oesophagitis OR esophagitis OR GERE OR Gastroesophageal reflux disease OR reflux esophagitis OR reflux esophagitis "," Children "," Proton pump inhibitor OR omeprazole "," Antihistamine $\mathrm{H} 2$ or ranitidine antagonists "for search in Indonesian. The inclusion criteria were articles of systematic review, randomized controlled clinical trials and cohort studies, with the limitations of the study being carried out on humans, the subject of research was children and the language of instruction was English or Indonesian. The level of evidence (LOE) is determined based on the classification issued by the Oxford Center for Evidence-Based Medicine in 2011.

Table.1 Literature search results

\begin{tabular}{|c|c|c|c|}
\hline & Search Method & $\begin{array}{c}\text { Number of } \\
\text { articles obtained }\end{array}$ & $\begin{array}{c}\text { Relevant } \\
\text { articles }\end{array}$ \\
\hline Pubmed & $\begin{array}{l}\text { (((((() (reflux oesophagitis) OR (esophagitis)) OR (GERD)) } \\
\text { OR (gastroesophageal reflux disease)) OR } \\
\text { (gastroesophageal reflux)) OR (reflux esophagitis)) } \\
\text { AND (((children [MeSH Terms]) OR (pediatric [MeSH } \\
\text { Terms])) OR (childhood [MeSH Terms]))) AND ((proton } \\
\text { pump inhibitors [MeSH Terms]) OR (omeprazole [MeSH } \\
\text { Terms]))) AND ((ranitidine [MeSH Terms]) OR (H2 } \\
\text { antihistamine antagonist)) }\end{array}$ & 82 & 6 \\
\hline
\end{tabular}




\begin{tabular}{|c|c|c|c|}
\hline Google Scholar & $\begin{array}{l}\text { (((()((reflux oesophagitis) OR (esophagitis)) OR (GERD)) } \\
\text { OR (gastroesophageal reflux disease)) OR } \\
\text { (gastroesophageal reflux)) OR (reflux esophagitis)) } \\
\text { AND (((children [MeSH Terms]) OR (pediatric [MeSH } \\
\text { Terms])) OR (childhood [MeSH Terms]))) AND ((proton } \\
\text { pump inhibitors [MeSH Terms]) OR (omeprazole [MeSH } \\
\text { Terms]))) AND ((ranitidine [MeSH Terms]) OR (H2 } \\
\text { antihistamine antagonist)) }\end{array}$ & 149 & 6 \\
\hline Cochrane & $\begin{array}{l}\text { (((()((reflux oesophagitis) OR (esophagitis)) OR (GERD)) } \\
\text { OR (gastroesophageal reflux disease)) OR } \\
\text { (gastroesophageal reflux)) OR (reflux esophagitis)) } \\
\text { AND (((children [MeSH Terms]) OR (pediatric [MeSH } \\
\text { Terms])) OR (childhood [MeSH Terms]))) AND ((proton } \\
\text { pump inhibitors [MeSH Terms]) OR (omeprazole [MeSH } \\
\text { Terms]))) AND ((ranitidine [MeSH Terms]) OR (H2 } \\
\text { antihistamine antagonist)) }\end{array}$ & 81 & 0 \\
\hline $\begin{array}{l}\text { Pediatrica } \\
\text { Indonesiana }\end{array}$ & $\begin{array}{l}\text { reflux oesophagitis OR esophagitis OR GERD OR } \\
\text { gastroesophageal reflux disease OR } \\
\text { gastroesophageal reflux OR reflux esophagitis AND } \\
\text { Children OR pediatric AND Proton pump inhibitor OR } \\
\text { omeprazole AND Antagonist Antihistamine H2 OR } \\
\text { ranitidine. }\end{array}$ & 0 & 0 \\
\hline Sari Pediatrics & $\begin{array}{l}\text { Reflux oesophagitis OR esophagitis OR GERE OR } \\
\text { Gastroesophageal reflux disease OR reflux } \\
\text { esophagitis "AND Children AND Proton pump } \\
\text { inhibitors OR omeprazole AND H2 antihistamine or } \\
\text { ranitidine antagonists }\end{array}$ & 0 & 0 \\
\hline
\end{tabular}

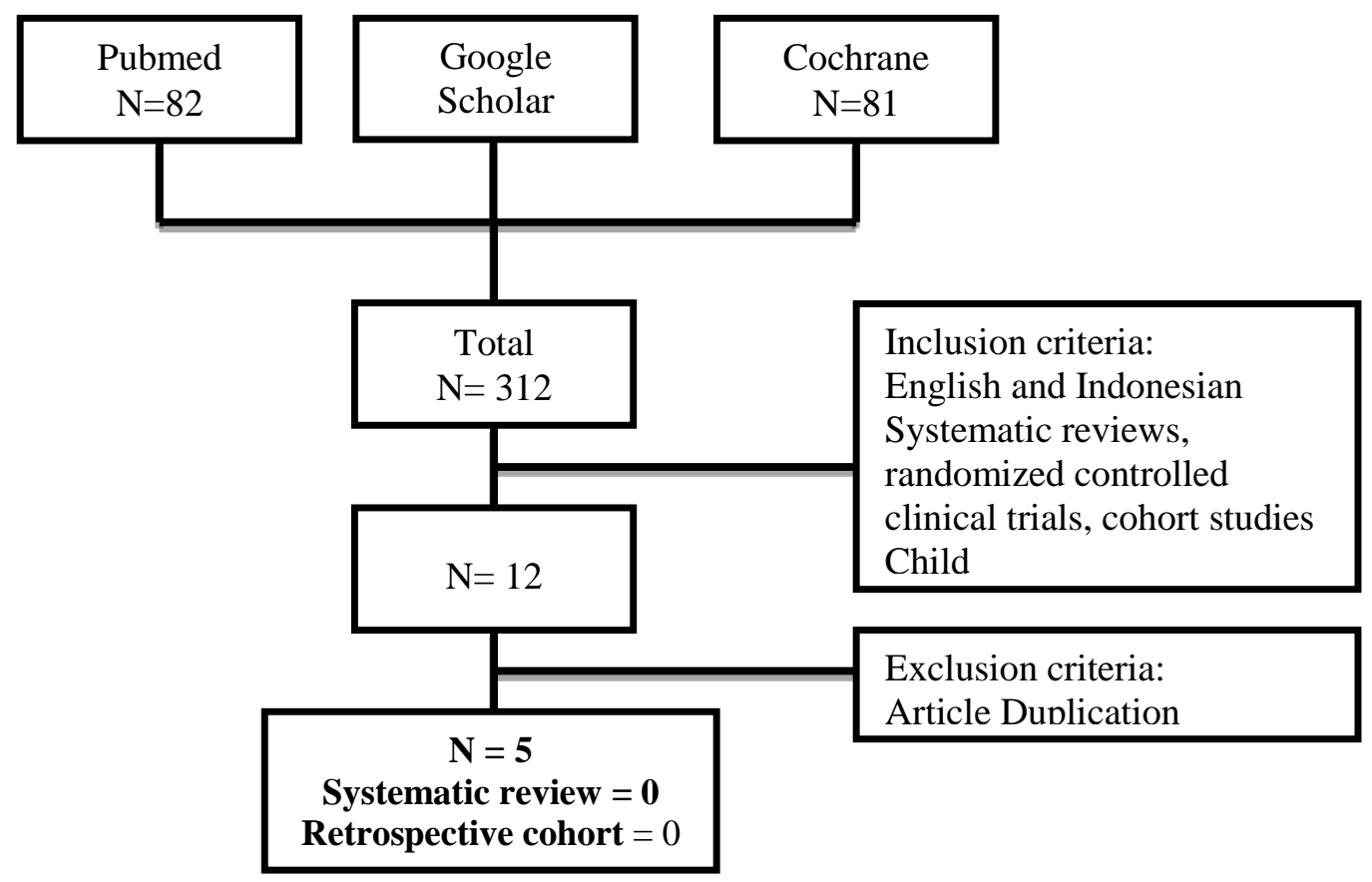

Figure 1. Article Search Flow

\section{Result}

A search of the articles in this paper found five relevant articles consisting of three studies comparing ranitidine and omeprazole in children with new cases of GERD and two studies that each compared the effectiveness of the two drugs in preventing recurrence 
of symptoms and incidence of nocturnal acid breakthrough. Here are three studies that explain the comparison of the two drugs in overcoming GERD symptoms:

The following two studies did not examine untreated
GERD, but each compared the effectiveness of the two drugs (ranitidine and omeprazole) in preventing recurrence of symptoms and preventing nocturnal acid breakthrough in patients who had received treatment and experienced improvement including:

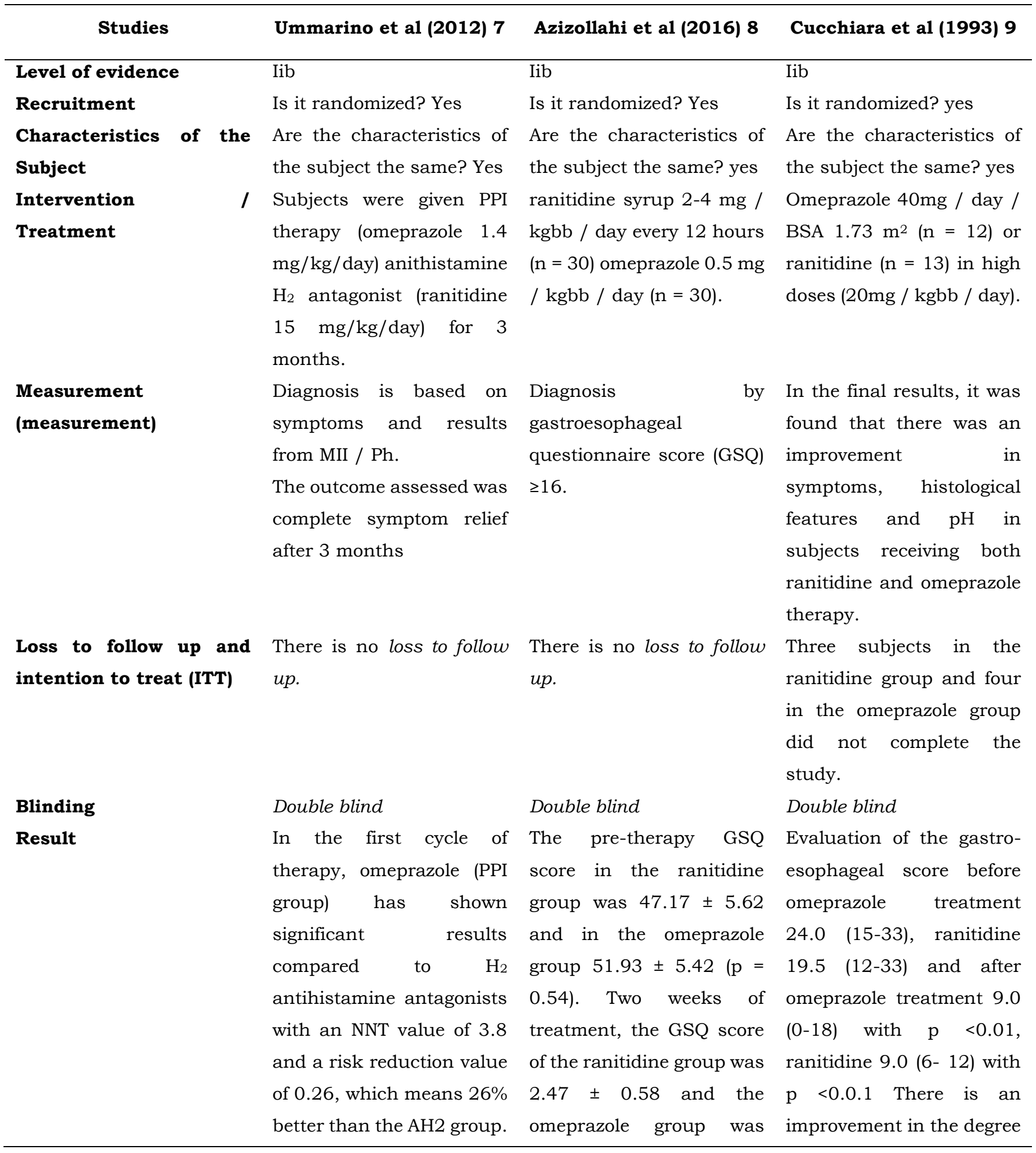


Outcome

Complete

Results symptom

resolution was found in

$83 \%$ of subjects receiving

PPIs and $35.3 \%$ of subjects

(OR 8.8; $\mathrm{P}=0.03)$, with an

NNT of 2.08 .
$2.43 \pm 1.15$ after therapy $(\mathrm{p}=0.98)$. There is no explanation regarding the side effects in this study. of histology and a decrease in the duration of reflux.

Boccia et al (2007) ${ }^{11}$

Pfefferkorn et al (2006) ${ }^{10}$

Level of evidence

Recruitment

Characteristics of the Subject

Intervention / Treatment

Measurement (measurement)

(ntervention $/$ Treatment

Iib

Iib

Is it randomized? yes

Are the characteristics of the subject the same? Yes PPI + ranitidine versu placebo.
Measurement (measurement)
Clinical, histological, endoscopic criteria
Iib

Is it randomized? Yes

Are the characteristics of the subject the same? yes

The initial study conducted was to provide initial therapy of omeprazole at a dose of 1.4 $\mathrm{mg} / \mathrm{kg} /$ day for 3 months. Patients in endoscopic remission were randomly assigned, blinded using a computerized general list to three 6-month maintenance treatment groups: group A (omeprazole at half the initial dose, once daily before breakfast), group B (ranitidine 10 $\mathrm{mg} / \mathrm{kg} /$ day, divided in two doses), and group $\mathrm{C}$ (not receiving treatment).

and Endoscopic, histologic, and symptom scores were evaluated at: T0, enrollment; T1, assessment for remission at 3 months after admission (healing phase); T2, the assessment for maintenance is effective at 12 months after TO (3 months after the completion of the maintenance phase

Loss to follow up and intention to Two subjects in the ranitidine There is no loss to follow up. 
treat (ITT)

\section{Blinding}

Result group and one in the placebo

group did not complete the study

Double blind

Nocturnal acid breakthrough

occurred in $75 \%$ of PPI +

ranitidine subjects and $75 \%$ of

PPI + placebo subjects. However,

there was a significant

improvement in the patient's

endoscopy and histology (p

$<0.05$ )

\section{Double blind}

Only 1 case of relapse was in the non-therapy group. There was no statistically significant difference between the three groups

\section{Discussion}

Gastroesophageal reflux is a physiological process that usually occurs in the first 1 year of life. Gastroesophageal reflux disease is described as RGE that causes symptoms, impaired quality of life to severe complications. ${ }^{1,5}$ The incidence of GERD is less common than RGE, but it requires medical therapy. The current recommendation is that getting the clinical history is very reliable in diagnosing GERD. History and examination are important to differentiate RGE and GERD and identify complications that may arise. ${ }^{12}$

Symptoms and signs associated with GERD in infants and children are divided into general symptoms, gastrointestinal symptoms and respiratory symptoms.1,2 Common symptoms and signs include irritability, failure to thrive, refusal to eat, Sandifer position (dystonic neck), tooth erosion. and anemia. Gastrointestinal symptoms that can be found are recurrent regurgitation with or without vomiting in older children, heartburn or chest pain, epigastric pain, hematemesis, dysphagia or odynophagia. Signs of esophagitis, esophageal strictures, and esophageal barrettes can be found on investigations. Symptoms and signs of the respiratory system that can be found are wheezing, stridor, coughing, hoarseness, apneic attacks (in neonates), asthma, recurrent pneumonia and recurrent otitis media. esophageal biopsy and monitoring of esophageal pH.5,12,14

The main goals of management in GERD patients are to relieve symptoms, achieve optimal growth, and prevent complications. Gastroesophageal reflux disease requires medical therapy, especially drugs that suppress or inhibit gastric acid secretion. ${ }^{5}$ Effective and safe management to treat symptoms and signs of GERD can be done with non-pharmacological, pharmacological and surgical management. Nonpharmacological management of GERD such as thickening feeding, positioning, lifestyle modification such as avoiding alcohol and cigarette smoke. The recommended position is the left lateral position and head elevation which have been shown to reduce the symptoms of crying or breath distress in infants with GERD symptoms. ${ }^{5,15}$

Pharmacological management of GERD in children varies. The effectiveness of various types of drugs in overcoming the manifestations of GERD in children is still controversial. Various types of drugs that can be used, including antacids and alginates (neutralizing stomach acid), gastric acid suppressants such as PPIs and $\mathrm{H}_{2}$-receptor antagonists (functions to suppress stomach acid production), prokinetic groups (reduce acid reflux and accelerate gastric emptying), antidopaminergic groups such as domperidone and metoclopramide (facilitates gastric emptying), other prokinetic groups such as cisapride, erythromycin, and bethanicol. However, its use in children with GERD is controversial because no studies have assessed its effectiveness and safety. Currently recommended for children with GERD are antacids and gastric acid suppressants (PPIs and $\mathrm{H}_{2}$ receptor antagonists). ${ }^{2,5,6}$ 
Omeprazole substituted benzimidazole 5-Methoxy-2

[[(4-methoxy-3,5-dimethyl-2-pyridinyl) methyl] sulfinyl] $-1 \mathrm{H}$ benzimidazole is a class of proton pump inhibitors that reduce gastric acid secretion. Omeprazole blocks the proton pump in gastric parietal cells $(\mathrm{H}+\mathrm{K}+$-ATPase $)$ on the basolateral membrane, reduces basal gastric acid secretion and decreases its stimulation and increases intragastric $\mathrm{pH}$. Omeprazole has a good tolerance, for children who have difficulty swallowing the capsule, the capsule can be opened and the granule content in it can be given by mixing it in a moderately acidic liquid such as orange juice, cranberry juice or yogurt. Granules are stable in acidic conditions, but will degrade in neutral or alkaline conditions. ${ }^{16}$ Another anti-secretory drug, ranitidine, as an antihistamine $\mathrm{H}_{2}$ antagonist, acts by reducing histamine, which induces gastric acid secretion and pepsin production. Histamine released from enterochromaffin cells binds to receptors in parietal cells and plays a role in increasing cylic adenosine monophosphate and activating proton pumps. ${ }^{2,6}$

Surgery can be performed for cases of GERD with life-threatening complications (after failing with optimal drug therapy), or for chronic cases that cause GERD to continue, such as in conditions of neurological disorders, cystic fibrosis, and conditions that require chronic pharmacological therapy to control. Signs and symptoms of GERD. 5

Although the effectiveness of PPI drugs is still being debated, the use of PPIs in toddlers and children in the last decade has increased. Several studies that have been done have stated that PPIs are effective in GERD therapy with minimal side effects. PPIs significantly improve heartburn symptoms compared to $\mathrm{H}_{2}$ antihistamine antagonists. Omeprazole as one of the PPI classes is effective for the treatment of recurrent and severe childhood RGE. ${ }^{8}$ On the other hand, several studies have stated that $\mathrm{H}_{2}$ antihistamine antagonists are less effective in curing esophagitis because they are not effective in inhibiting foods that stimulate acid secretion. 14
In the case illustration above, there are five studies that are relevant to answer the clinical questions of the case. In general, the evidence regarding GERD treatment in children is still lacking. Although there are three RCT studies comparing the effectiveness of omeprazole and ranitidine in the treatment of GERD, the three studies have various differences. The dosage of drugs given in each study was not the same, especially the dose of ranitidine which could range from 2-20mg / kgbb / day. The diagnostic criteria used also differed between studies. The outputs used in each study also differed between clinical symptoms, microscopic appearance on histopathological biopsy, macroscopic endoscopy, and esophageal $\mathrm{pH}$ measurements. Few studies have conducted long-term evaluations of GERD treatment.

One study by Azizollahi et al in infants with GERD showed that after 2 weeks of standard doses of omeprazole or ranitidine (2-4 mg / kg / day) there was significant and comparable improvement in the two groups. This result is different from the study of Ummarino et al, which showed that omeprazole was significantly better than high-dose ranitidine $(15 \mathrm{mg} /$ kg / day). Research conducted by Azizollahi et al. Was conducted on infants, while Umarino et al's study was conducted on children aged $40.6 \pm 36.4$ months. The diagnostic criteria for Azizollahi et al's study only used clinical scoring, while Ummarino et al's study used $\mathrm{pH}$ monitoring. The difference in outcome may also be due to differences in the success of therapy, in the study of Azizollahi et al. It was an improvement in symptoms but in the study of Ummarino et al. It was characterized by complete symptom relief. The follow-up in Azizollahi et al's study lasted only two weeks while Ummarino et al's study was conducted for 3-6 months. Based on these two studies, it can also be raised a suspicion whether ranitidine is more effective in infants than in older children, although we have not found any publications that support this. Interestingly, another study by Cucchiara et al (1993) showed that high-dose ranitidine $(20 \mathrm{mg} / \mathrm{kgbb} /$ day $)$ was as good as omeprazole in terms of improving clinical, histological 
features, endoscopy, and $\mathrm{pH}$ monitoring in 32 children aged 6 months-13, 4 years with GERD resistant to standard dose ranitidine $(8 \mathrm{mg} / \mathrm{kgbb} / \mathrm{day})$ and cisapride. Cucchiara et al's study used the most comprehensive outcome assessment compared to the other two studies. Although, the dose in the Cucchiara study was slightly higher than that in Ummarino's study, there may indeed be a dose effect in terms of administering ranitidine to children with GERD. Neither the standard nor the high dose administration of ranitidine have been associated with serious side effects although none of the three publications provide details on this.

There are two other studies by Pfefferkorn et al. And Boccia et al. Which examined nocturnal acid breakthrough and esophagitis in children who had previously received omeprazole therapy. Pfefferkorn et al's study compared the combination of ranitidine and omeprazole versus omeprazole and placebo in preventing the incidence of TLV. The results showed that the incidence of TLV was very high in both groups (75\%). However, there was still significant improvement in clinical and histological symptoms. Boccia et al's study compared omeprazole, ranitidine, and nontherapy in preventing GERD relapse after 3 months of omeprazole treatment. There was a very low and insignificant relapse rate when the three groups were compared.

\section{Conclusion}

Evidence regarding the use of ranitidine versus omeprazole in infants and children is lacking. Based on one study specifically in the infant age group, omeprazole and ranitidine had comparable effectiveness in improving clinical symptoms of GERD. A higher dose of ranitidine may have a better effect. In terms of complete symptom relief, omeprazole is likely to be superior to ranitidine.

\section{Suggestion}

Based on a review of the evidence that has been carried out for pediatric patients aged 5 months with GERD, administration of a standard dose of ranitidine (2-4 mg/kgbb/day) can be done if there is difficulty in administering omeprazole. If an adequate response has not been obtained, an increase in the dose of ranitidine to $20 \mathrm{mg} / \mathrm{kg} /$ day can be considered. Administration should be continued for up to 3 months because it is associated with a low relapse rate.

\section{REFERENCES}

1. Winter H, Gunasekaran T, Tolia V, Gottrand F, Barker PN, Illueca M. Esomeprazole for the Treatment of GERD in Infants Ages 1-11 Months. J Pediatr Gastroenterol Nutr. 2015;60:S9-S15.

2. Tighe MP, Afzal NA, Bevan A, Beattie RM. Current pharmacological management of gastroesophageal reflux in children: An evidence-based systematic review. Pediatr Drugs. 2009;11(3):185202 .

3. Diaz DM, Winter HS, Colletti RB, dkk. Knowledge, attitudes and practice styles of North American pediatricians regarding gastroesophageal reflux Disease. J Pediatr Gastroenterol Nutr. 2007;45(1):56-64.

4. El-Serag HB, Sweet S, Winchester CC, Dent J. Update on the epidemiology of gastro-oesophageal reflux disease: A systematic review. Gut. 2014;63(6):871-80.

5. Vandenplas Y, Rudolph CD, Di Lorenzo C, dkk. Pediatric Gastroesophageal Reflux Clinical Practice Guidelines: Joint Recommendations of the North American Society for Pediatric Gastroenterology, Hepatology, and Nutrition (NASPGHAN) and the European Society for Pediatric Gastroenterology, Hepatology, and Nutrition (ESPGHAN). J Pediatr Gastroenterol Nutr. 2009;49(4):498-547.

6. de Mattos ÂZ, Marchese GM, Fonseca BB, Kupski C, Machado MB. Antisecretory treatment for pediatric gastroesophageal reflux disease - A 
systemic review. Arq Gastroenterol. 2017;54(4):271-80.

7. Ummarino D, Miele E, Masi P, Tramontano A, Staiano A, Vandenplas Y. Impact of antisecretory treatment on respiratory symptoms of gastroesophageal reflux disease in children: PPI in extraesophageal GERD symptoms. Dis Esophagus. 2012;25(8):671-77.

8. Azizollahi HR, Rafeey M. Efficacy of proton pump inhibitors and $\mathrm{H} 2$ blocker in the treatment of symptomatic gastroesophageal reflux disease in infants. Korean J Pediatr. 2016;59(5):226. doi:10.3345/kjp.2016.59.5.226

9. Cucchiara S, Minella R, Iervolino C, dkk. Omeprazole and high dose ranitidine in the treatment of refractory reflux oesophagitis. Arch Dis Child. 1993;69(6):655-59.

10. Pfefferkorn MD, Croffie JM, Gupta SK, dkk. Nocturnal acid breakthrough in children with reflux esophagitis taking proton pump inhibitors: J Pediatr Gastroenterol Nutr. 2006;42(2):160-65.

11. Boccia G, Manguso F, Miele E, Buonavolontà R, Staiano A. Maintenance Therapy for erosive esophagitis in children after healing by omeprazole: Is it advisable? Am J Gastroenterol. 2007;102(6):1291-297.

12. Salvatore S, Hauser B, Vandemaele K, Novario R, Vandenplas Y. Gastroesophageal reflux disease in infants: How much is predictable with questionnaires, pH-metry, endoscopy and histology? J Pediatr Gastroenterol Nutr. 2005;40(2):210-15.

13. Deal L, Gold BD, Gremse DA, dkk. Age-specific questionnaires distinguish GERD symptom frequency and severity in infants and young children: Development and initial validation. J Pediatr Gastroenterol Nutr. 2005;41(2):178-85.

14. Wang WH. Head-to-head comparison of $\mathrm{H} 2$ receptor antagonists and proton pump inhibitors in the treatment of erosive esophagitis: A meta- analysis. World J Gastroenterol. 2005;11(26):4067. doi:10.3748/wjg.v11.i26.4067

15. Loots C, Kritas S, van Wijk M, dkk. Body positioning and medical therapy for infantile gastroesophageal reflux symptoms. J Pediatr Gastroenterol Nutr. 2014;59(2):237-43.

16. Messaouik D, Sautou-Miranda V, Bagel-Boithias S, Chopineau J. Comparative study and optimisation of the administration mode of three proton pump inhibitors by nasogastric tube. Int $\mathrm{J}$ Pharm. 2005;299(1-2):65-72. 